\title{
The effects of temporal waveform upon temporal darkness enhancement
}

\author{
FREDERICK L. KITTERLE and HENRY C. TONEY, JR. \\ University of Toledo, Toledo, Ohio
}

\begin{abstract}
Temporal darkness enhancement refers to the finding that decremental flashes of 50 $140 \mathrm{msec}$ appear darker than longer flash decrements. The present experiment determined the effects of temporal waveform upon darkness enhancement by obtaining darkness judgments for flashes that had abrupt onset/abrupt offset, abrupt onset/gradual offset, gradual onset/abrupt offset, and gradual onset/gradual offset. Temporal darkness enhancement was found only for flashes that had abrupt onsets regardless of offset waveform. These results are discussed in terms of the role of transients in the coding of perceived darkness.
\end{abstract}

A large number of psychophysical studies have been concerned with attempts to understand the nature of the mechanisms that underlie the subjective appearance of a temporally modulated target. Recent experiments have determined this by measuring the brightness of the bright phases and the darkness of the dark phases of a flickering light as a function of temporal frequency (Glad \& Magnussen, 1972; Magnussen \& Bjorklund, 1979; Magnussen \& Glad, 1975a, 1975b). Two related phenomena have been found. At flicker rates of $5-7 \mathrm{~Hz}$, the brightness (darkness) of the light (dark) phases is greater than steady light (dark). The former is referred to as "temporal brightness enhancement," and the latter, as "temporal darkness enhancement." Generally, the magnitude of darkness enhancement is larger than that of brightness enhancement (Glad, Magnussen, \& Engvik, 1976; White, Irvin, \& Williams, 1980). These results are interesting in light of theoretical evidence suggesting the existence of two functionally separate and complementary neural subsystems, a B system that signals the brightness of a target and a D system that signals darkness (Jung, 1973; Grind, Grusser, \& Lunkenheimer, 1973). Jung (1973) has suggested that these two systems have electrophysiological correlates in the activity of on-center neurons in the case of the B system and offcenter neurons in the case of the $D$ system, and that these neural systems are independent and reciprocally organized.

The sensation of brightness or darkness of a flash depends upon some feature of a neural response which varies in time from the onset of the flash. In general, there is a large burst of activity which accompanies the onset and offset of a flash (Kuffler, 1953). It has been suggested that the initial transient

The authors' mailing address is: Department of Psychology, University of Toledo, Toledo, Ohio 43606. associated with the onset of a luminance increment is utilized in the coding of brightness (Adrian, 1928). One way of determining the role of response transients in sensory coding is to observe the effects of varying their magnitude upon sensation. Recent psychophysical techniques indicate that variations in temporal waveform (the rate at which a target is turned on or off) can influence the magnitude of response transients (Breitmeyer \& Julesz, 1975; Matsumura, 1976). Transient activity decreases as the onsets and offsets are made more gradual. Thus, variations in temporal waveform may provide information about the features of a neural response utilized by these subsystems in coding brightness and darkness. If transients play a role in sensory coding, then the rate at which a target is turned on and off may influence its perceived brightness and darkness. Recently, Kitterle and Corwin (1983) found that temporal brightness enhancement occurs only when the onset of a target is abrupt regardless of the offset waveform. This result suggests that the neural activity associated with the onset of a target (the socalled "on-response") may be more important in the coding of brightness than the neural activity associated with the offset (Boynton \& Kandel, 1957; Ikeda \& Boynton, 1965; Onley \& Boynton, 1962).

Little is known about the effects of temporal waveform upon darkness enhancement. However, if the B system complements the D system (Jung, 1973), then the results of Kitterle and Corwin (1983) suggest that the initial neural activity associated with a luminance decrement (the so-called "off-response") is necessary for obtaining darkness enhancement. Such a hypothesis would imply that the initial transient is the crucial neural feature utilized by the visual system in the coding of either the brightness or darkness of brief flashes $(50-120 \mathrm{msec})$. Thus, reducing the magnitude of the off-response should abolish temporal darkness enhancement. To test this hypothesis, perceived darkness was measured as a function of flash 
duration under one of four temporal waveforms: abrupt onset/abrupt offset, abrupt onset/gradual offset, gradual onset/abrupt offset, and gradual onset/gradual offset. The results of this experiment supported the above idea; temporal darkness enhancement was found only when the luminance decrement had an abrupt onset.

\section{METHOD}

\section{Observers}

Forty-four University of Toledo undergraduates served in this experiment for course credit. They were assigned randomly to one of four temporal waveform conditions using 11 observers each. All observers had normal or corrected-to-normal vision, and they were naive about the purpose of this experiment.

\section{Apparatus}

A two-channel haploscopic system was used to present a dark vertical bar to each eye. The stimuli were generated on two separate oscilloscopes (Tektronix 5103/DN, P-31 phosphor), using conventional techniques. When dim red fixation points mounted on the side of each oscilloscope were fused, the display consisted of a single fixation point between two circular fields which, at a viewing distance of $127 \mathrm{~cm}$, subtended a visual angle of $3.7 \mathrm{deg}$. The targets appeared spatially adjacent and separated by 3.4 deg. The width of the vertical bars was $1.4 \mathrm{deg}$. The bars were produced by decrementing the luminance of the central portion of each aperture. The luminance of the decrement was $1.70 \mathrm{fL}$ and that of the background, $1.92 \mathrm{fL}$, as determined by an EG\&G 550 photometer/radiometer. A SYM-1 microprocessor controlled timing and data collection.

\section{Procedure}

Observers were assigned randomly to one of four experimental conditions, which consisted of abrupt onset/abrupt offset, abrupt onset/gradual offset, gradual onset/abrupt offset, or gradual onset/gradual offset. In the gradual on- and offset conditions, the rise-and-fall time of the target luminance was $20 \mathrm{msec}$. In an experimental session, the observer initially adapted to the display luminance for $2 \mathrm{~min}$, during which instructions were read. After the adaptation period, both bar targets. were presented and the observer adjusted the darkness of one bar until it was perceived to be equal in darkness to the other. Following this, the experimental session began. Initially, there were a number of practice trials in order to familiarize observers with the procedure.

Each trial consisted of the presentation of a short pulse and a long pulse, of the same temporal waveform, with simultaneous offset. At any given duration, all temporal waveforms were equated in energy. The long pulse was $500 \mathrm{msec}$, while the short pulse varied in duration. Darkness judgments were made for 11 pulse durations. The observers participated in two sessions, one consisting of 20 replications of six different pulse durations and the other of 20 replications of five different pulse durations, which were randomly ordered and randomly presented to either the left or right eye. Typically, the intertrial interval lasted $7 \mathrm{sec}$. Each experimental session lasted about $25 \mathrm{~min}$.

The format used to display the data is similar to that of Bowen and Pokorny (1978). The percent of time the longer flash is judged darker is plotted as a function of the duration of the shorter flash. For the range of very brief to intermediate-duration flashes, in which darkness increases with duration, the longer flash should always be judged darker than the shorter flash. If there is darkness enhancement, then there will be a shorter flash duration in which the longer flash will never be judged darker. Finally, in the range in which darkness is independent of duration, the longer flash should be judged darker than the shorter flash approximately $50 \%$ of the time.

\section{RESULTS}

The results of this experiment are shown in Figure 1 . The symbols represent the following conditions: abrupt onset/abrupt offset (filled circles), abrupt onset/gradual offset (unfilled circles), gradual onset/abrupt offset (unfilled squares), and gradual onset/gradual offset (filled squares).

The filled and open circles fall significantly below $50 \%$ by the binomial test based upon average data. This indicates the presence of a darkness-enhancement effect within the range of $160-200 \mathrm{msec}$ for both the abrupt onset/abrupt of fset and the abrupt onset/ gradual offset conditions. A secondary enhancement effect was also found at $360 \mathrm{msec}$ with the abrupt onset/abrupt offset condition. Although the data for the ramped conditions (filled and open squares) fall below $50 \%$ over the range of $200-360 \mathrm{msec}$, these results are not significant. Thus, an abrupt onset seems to be a necessary condition for obtaining temporal

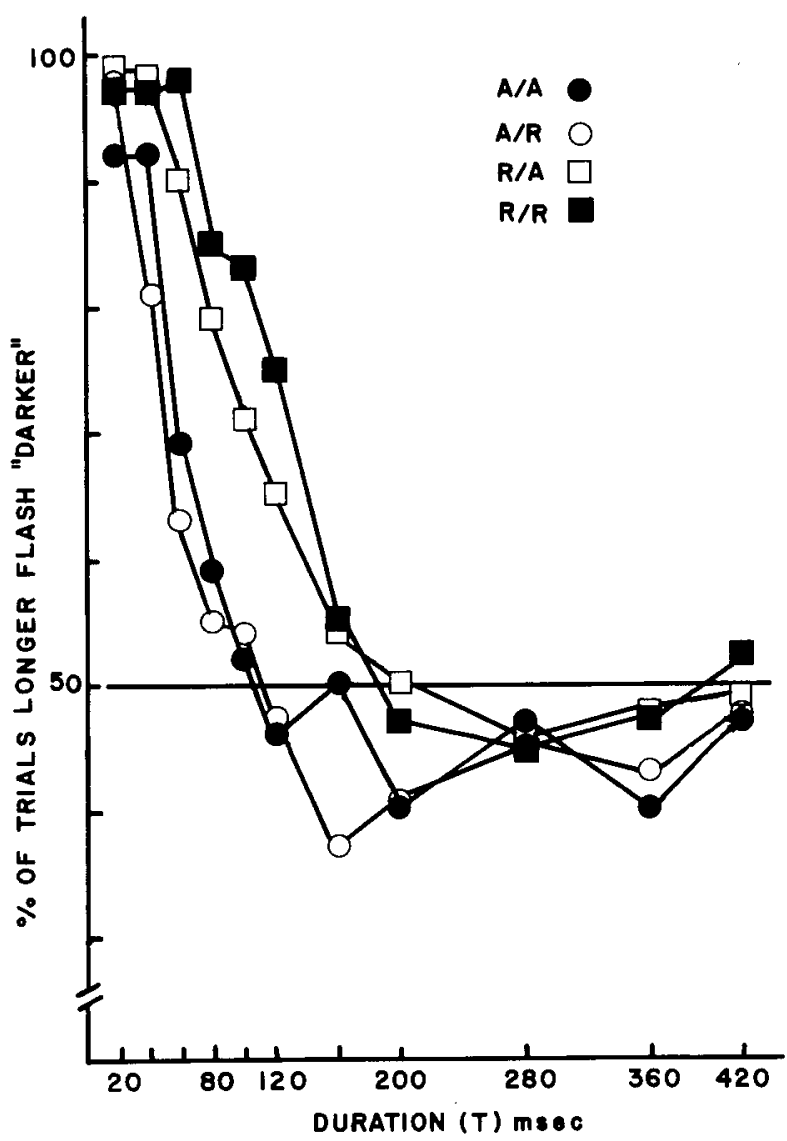

Figure 1. Percentage of time a longer (500-msec) flash was judged darker than fashes of a shorter duration. The symbols indicate the following temporal waveforms: abrupt onset/abrupt offset (filled circles), abrupt onset/gradual offset (open circles), gradual onset/abrupt offset (unfilled squares), and gradual onset/gradual offset (filled squares). Each data point is the average of 220 observations. 
darkness enhancement. Furthermore, the offset does not seem to influence the effect. This conclusion is confirmed by a supplemental analysis.

The data were analyzed in a one-between (temporal waveform) and one-within (pulse duration) factor analysis of variance. There were significant main effects of temporal waveform $[F(3,40)=7.69$, $\mathrm{p}<.01]$ and flash duration $[\mathrm{F}(10,400)=109.82$, $\mathrm{p}<.01]$. This analysis indicated a significant interaction between temporal waveform and flash duration $[F(30,400)=3.46, p<.01]$. A fine-grain analysis revealed the source of the differential effect of temporal waveform across duration. The abrupt onset conditions vs. the gradual onset conditions were compared across flash duration and found to be significant $[F(10,420)=8.44, p<.01]$. Neither the comparison between the gradual onset/abrupt offset vs. the gradual onset/gradual offset across duration nor the abrupt onset/gradual offset vs. the abrupt onset/abrupt offset across duration proved to be significant.

A further comparison of the results obtained with ramped (squares) and abrupt onset (circles) conditions indicates that the ramped data cross the $50 \%$ line at $\mathbf{2 0 0} \mathrm{msec}$ while the abrupt data cross this line at $100 \mathrm{msec}$. Since gradual onsets reduce the magnitude of the onset transient, this result complements Legge's (1978) findings that the limits of temporal summation increase when onset transients are reduced.

\section{DISCUSSION}

The results of this experiment indicate that when response transients associated with a decrement in luminance are reduced, temporal darkness enhancement is abolished. The data shown in Figure 1 also indicate that temporal offset does not influence this effect. Kitterle and Corwin (1979) suggest that transient neural activity is necessary for obtaining temporal brightness enhancement. A later paper (Kitterle \& Corwin, 1983) has shown that temporal contrast enhancement is abolished by ramping on a target, and Kitterle and Beard (1983) have found that flicker adaptation can abolish temporal contrast enhancement. Flicker adaptation appears to decrease transient activity at suprathreshold levels (Breitmeyer, Levi, \& Harwerth, 1981). These results provide strong support for the conclusion that transient activity is a necessary condition for temporal contrast enhancement. The present results extend that conclusion by showing transient activity associated with the onset of a target to be necessary for obtaining temporal darkness enhancement. These studies emphasize the role of transients in the coding of sensory magnitude and contradict earlier conclusions suggesting that the correlation between transient neural activity and temporal brightness enhancement is illusory (Wasserman \&
Kong, 1974). Wasserman and Kong (1974) reject the idea that neural transients are necessary for temporal brightness enhancement because brightness changes in a nonmonotonic fashion with duration while neural transients appear to change in a monotonic manner with duration.

Although we have shown that transient activity is necessary for obtaining temporal darkness enhancement, it is difficult to understand how a sensory magnitude is computed for a flash of a given duration, because we do not know how sensation changes as a function of time for a flash of a given duration. It may be that transient activity is important in the coding of the sensory magnitude of flashes of intermediate and short duration, but that it plays a diminished role in the coding of the brightness of longduration flashes. For example, Barlow and Verrillo (1976) have shown that there is no difference in the brightness judgments of long-duration flashes which differ in onset rise time.

The criteria upon which brightness or darkness is based may change as a function of exposure duration. Perhaps observers in the Barlow and Verrillo (1976) study based their brightness judgments upon the neural activity toward the end of the flash. Observers may also differ in the criteria used to code the brightness of a flash of a given duration (Bowen \& Markell, 1980; Bowen, Sekuler, Owsley, \& Markell, 1982), or stimulus conditions may favor the adoption of one criterion over another (Raab \& Osman, 1962). For example, Bowen and Markell (1980) found a temporal brightness-enhancement effect for some observers when the flashes had either simultaneous onset or simultaneous offset (Class A). Other observers showed the effect only if the flashes had simultaneous offset (Class B). A third group (Class C) did not show this effect for either asynchrony condition. Bowen and Markell (1980) suggest that observers differ in terms of whether they base the brightness response upon peak sensory activity, a temporal integral of sensory activity, or sustained activity. Raab and Osman (1962) have shown that the perceived brightness of a flash depends upon its onset relative to that of a standard. The brightness obtained when the flashes have simultaneous onset differs from that obtained when the two have simultaneous offsets. This is consistent with the idea that the simultaneous onset condition emphasizes a comparison of the transient activity of the two flashes, whereas the simultaneous offset condition emphasizes a comparison of the sustained activity of one flash with the transient activity of the other.

In summary, the results of this study demonstrate that temporal darkness enhancement is obtained when flashes have abrupt onsets. Darkness enhancement is not found when the flashes have a gradual onset, and the way in which a flash is turned off does not influence the effect. These results indicate that 
the transient activity that accompanies the onset of a target plays an important role in the coding of the darkness of intermediate-duration flashes. Other studies, however, suggest that transient activity may be necessary but not sufficient for obtaining temporal enhancement effects. Observers may differ in the criteria used to code brightness and darkness.

\section{REFERENCES}

Adnian, E. D. The physical basis of sensation: The action of sense organs. London: Christophors, 1928.

BARLOW, R. B., \& VERRILLo, R. Brightness sensation in a ganzfeld. Vision Research, 1976, 16, 1291-1298.

Bowen, R. W., \& MarkelL, K. A. Temporal brightness enhancement studied with a large sample of observers: Evidence for individual differences in brightness perception. Perception \& Psychophysics, 1980, 27, 465-476.

Bowen, R. W., \& Pokorny, J. Target edge sharpness and temporal brightness enhancement. Vision Research, 1978, 18, 1691-1695.

Bowen, R. W., Sekuler, R., Owsley, C. J., \& Markell, K. A. Individual differences in pulse brightness perception. Perception \& Psychophysics, 1982, 30, 587-593.

Boynton, R. M., \& Kandel, G. On-responses of the human visual system as a function of adaptation level. Journal of the Optical Society of America, 1957, 47, 275-286.

Breitmeyen, B., \& Julesz, B. The role of on and off transients in determining the psychophysical spatial frequency response. Vision Research, 1975, 15, 411-415.

Breitmeyer, B., Levi, D. M., \& Harwerth, R. S. Flicker masking in spatial vision. Vision Research, 1981, 21, 1377-1386.

Glad, A., \& Magnussen, S. Darkness enhancement in intermittent light: An experimental demonstration. Vision Research, 1972, 12, 111-115.

Glad, A., Magnussen, S., \& Engvik, H. Temporal brightness and darkness enhancement; further evidence for asymmetry. Scandinavian Journal of Psychology, 1976, 17, 234-237.

Grind, W. A. van de, Grusser, O. J., \& Lunkenheimer, H. U. Temporal transfer properties of the afferent visual system. In R. Jung (Ed.), Handbook of sensory physiology (Vol. VII/3A). New York: Springer, 1973.

IKedA, M., \& Boynton, R. M. Negative flashes, positive flashes and flicker examined by increment threshold technique.
Journal of the Optical Society of America, 1965, 55, 560-566. Jung, R. Visual perception and neurophysiology. In R. Jung (Ed.), Handbook of sensory physiology (Vol. VII/3A). New York: Springer, 1973.

KitTerle, F. L., \& Conwin, T. R. Enhancement of contrast in flashed sinusoidal gratings. Vision Research, 1979, 19, 33-39.

Kitterle, F. L., \& BeArD, B. L. The effects of flicker adaptation upon temporal contrast enhancement. Perception \& Psychophysics, 1983, 33, 75-78.

Kitterle, F. L., \& Conwin, T. R. The effects of temporal waveform upon apparent contrast. Perception \& Psychophysics, 1983, 33, 72-74.

KUFFLer, S. W. Discharge patterns and functional organization of mammalian retina. Journal of Neurophysiology, 1953, 16, 371-372.

LEgGE, G. Sustained and transient mechanisms in human vision: Temporal and spatial properties. Vision Research, 1978, 18, 69-81.

Magnussen, S., \& Bjorklund, R. A. The perception of suprathreshold sinusoidal flicker measured by light- and dark-phase matching. Vision Research, 1979, 19, 335-338.

Magnussen, S., \& Glad, A. Brightness and darkness enhancement during flicker: Perceptual correlates of neuronal B- and D-systems in human vision. Experimental Brain Research, 1975, 22, 399-413. (a)

Magnissen, S., \& Glad, A. Effects of steady surround illumination on the brightness and darkness enhancement of flickering lights. Vision Research, 1975, 15, 1413-1416. (b)

Matsumura, M. Visual masking by luminance increment and decrement: Effects of rise time and decay time. Tohoku Psychologia Folia, 1976, 35, 104-114.

Onley, J. W., \& Boynton, R. M. Visual responses to equally bright stimuli of unequal luminance. Journal of the Optical Society of America, 1962, 52, 934-940.

RAAB, D., \& Osman, R. Effect of temporal overlap on brightness matching of adjacent flashes. Journal of the Optical Society of America, 1962, 52, 1174-1178.

Wasserman, G. S., \& Kong, K. L. Illusory correlation of brightness enhancement and transients in the nervous system. Science, 1974, 184, 911-913.

White, T. W., Irvin, G. E., \& Williams, M. C. Aymmetry in the brightness and darkness Broca-Sulzer effects. Vision Research, 1980, 20, 723-726.

(Manuscript received September 13, 1982; revision accepted for publication November 18, 1982). 